\title{
Quantitative Sensory Testing in adults with Tourette syndrome
}

Odette Schunke ${ }^{1 *}$, Wiebke Grashorn ${ }^{1 *}$, Ursula Kahl ${ }^{1}$, Daniel Schöttle ${ }^{6}$, Patrick Haggard $^{2}$, Alexander Münchau ${ }^{3}$, Ulrike Bingel ${ }^{5}$, Christos Ganos ${ }^{1,2,4}$

*both authors contributed equally to this manuscript

1Department of Neurology, University Medical Center Hamburg-Eppendorf, Hamburg, Germany

${ }^{2}$ Institute of Cognitive Neuroscience, University College London, London, UK

${ }^{3}$ Department of Paediatric and Adult Movement Disorders and Neuropsychiatry, Institute of Neurogenetics, University of Luebeck, Luebeck, Germany

${ }^{4}$ Sobell Department of Motor Neuroscience and Movement Disorders, UCL Institute of Neurology Queen Square, London, UK

${ }^{5}$ Department of Neurology, University Hospital Essen, University Duisburg-Essen, Germany

${ }^{6}$ Department of Psychiatry, University Medical Center Hamburg-Eppendorf, Hamburg, Germany

\section{Corresponding Author:}

Odette Schunke

Department of Neurology, University Medical Center Hamburg-Eppendorf, Martinistraße 52, 20246 Hamburg, Germany

Email: o.schunke@uke.de; Telephone: 0049-40/ 7410-50134

Keywords: Tourette syndrome, quantitative sensory testing, premonitory sensations, sensory, thresholds

Word count: abstract 176 words, manuscript 2142 words 


\section{ABSTRACT}

Introduction: Abnormal sensory perceptions, for instance hypersensitivity to certain external stimuli or premonitory urges preceding tics, are core features in Gilles de la Tourette syndrome (GTS). Aberrant awareness of externally applied stimuli in terms of altered sensory perception thresholds might contribute to these sensory phenomena in GTS.

Methods: We used the well-established and standardized "Quantitative Sensory Testing" (QST) battery (German Research Network on Neuropathic Pain) to investigate 13 sensory parameters including thermal, mechanical/tactile and pain thresholds in 14 GTS patients without clinically significant comorbidities and 14 healthy controls matched for age and gender.

Results: There were no relevant group differences in any of the 13 QST parameters and no specific QST pattern in GTS patients. There was no correlation between QST parameters and "Premonitory Urge for Tics scale" (PUTS) scores.

Conclusion: Our data show that the perceptual threshold detection of externally applied sensory stimuli is normal in adults with GTS. This indicates that other perceptual mechanisms, such as abnormal central sensorimotor processing and/or aberrant interoceptive awareness might underlie the clinically significant sensory abnormalities in GTS. 


\section{INTRODUCTION}

Gilles de la Tourette syndrome (GTS) is a hyperkinetic movement disorder defined by the presence of multiple motor and phonic tics [1]. Tics differ from other hyperkinesias in that they share most neurophysiological and phenomenological properties of voluntary actions and can be inhibited on demand for variable periods of time. In the majority of GTS patients, particularly adults, tics are preceded by sensory phenomena referred to as premonitory sensations [2]. These aversive somatic experiences preceding the tic are often described rather abstract or generalized as an inner urge/impulse to tic, increased tension, anxiety or restlessness, but in some patients they may resemble rather localized somatic sensations such as ache, itching, tingling / burning, numbness or coldness [2]. Often, the execution of a tic gives relief to the preceding disturbing sensation [2].

It has been suggested that premonitory sensations could be the result of increased somatic sensitivity due to heightened perception of signals arising within (interoception) and/or outside the body (exteroception) [3, 4]. Indeed, for the interoceptive system, a recent study has provided first direct experimental evidence to link interoceptive awareness with premonitory sensations [5]. GTS adults who were better able to detect their heartbeats reported higher premonitory sensations to tic. However, the contribution of the exteroceptive system to tic-related sensory phenomena remains unclear. GTS patients are often hypersensitive towards particular external tactile stimuli (e.g. clothing tags, tight neckline) [4] and structural neuroimaging findings have demonstrated abnormalities within the primary somatosensory system [6]. Therefore, perception of externally applied stimuli (exteroception) might be altered 
in GTS patients and might contribute to the development of stimulus hypersensitivity and premonitory sensations.

To date, there have been only two attempts to evaluate sensory thresholds to external stimuli in GTS adults with mixed findings [4, 7]. However, the examined samples comprised patients with potentially confounding comorbidities, such as attention-deficit hyperactivity disorder (ADHD) and obsessive-compulsive behavior/disorder (OCD), and no more than two sensory modalities were assessed (olfactory [4] and tactile [4, 7]). Importantly, although both studies emphasized the role of the somatosensory system in premonitory urges, neither provided clinical information on their presence or their relation to sensory findings.

To further explore the potential contribution of the exteroceptive, but also nociceptive systems to tic-related sensory phenomena in GTS, we systematically assessed a battery of somatosensory functions by means of the established "Quantitative Sensory Testing" (QST) battery (German Research Network on Neuropathic Pain = "Deutscher Forschungsverbund Neuropathischer Schmerzen", DFNS) [8]. Our study sample comprised 14 well-characterized uncomplicated adults with GTS and without clinically significant comorbidities and age- and gender- matched healthy controls. Using QST information about peripheral (e.g. large and small fiber functions) and central mechanisms (e.g. sensitization/disinhibition) can be captured in addition to determining sensory thresholds.

Using this technique we aimed to obtaining potentially disease specific sensory profiles and to comparing them to age- and gender-matched healthy subjects and the DFNS reference values. Based on the patients' unusual sensory experiences (e.g. sensory 
hypersensitivity), we hypothesized reduced sensory thresholds, particularly to tactile/mechanical external stimuli. We further aimed at exploring the relationship between sensory thresholds and premonitory urges.

\section{METHODS}

\section{Participants}

The study was approved by the local ethics committee and was conducted in accordance with the Declaration of Helsinki. Written informed consent was obtained from all study participants who were told to be free to withdrawal from the study at any time. GTS participants were assessed in the outpatient clinic in the Department of Neurology, University Medical Center Hamburg-Eppendorf, Germany. GTS participants were diagnosed by neurologists specialized in movement disorders (A.M. or C.G.) using DSM-IV-TR criteria [1]. Only adult (>18 years) individuals with uncomplicated GTS (i.e. without ADHD or OCD) were included in the study to avoid confounding factors that might influence study results, for example inattention or OCDrelated sensory phenomena.

Adult healthy control subjects not taking any medication other than contraceptives were recruited locally and matched by age and gender. GTS patients without apparent comorbidity were recruited from the GTS clinics of one of the authors (A.M.). Subsequently, a semi-structured neuropsychiatric interview with a particular view to the DSM-IV-TR criteria of ADHD, OCD and depression was conducted (D.S., C.G.) and confirmed the absence of clinically relevant comorbidities. None of the subjects suffered from a chronic pain disorder.

\section{Questionnaires and Quantitative Sensory Testing (QST)}


Sensory phenomena were assessed based on Kwak et al. [2] and quantified by means of the German version of the Premonitory Urge for Tics Scale (PUTS [9]). QST was performed using an established battery and the standard equipment according to the DFNS [8]. All QST experimenters were specially trained by the DFNS to perform QST according to a standard protocol.

As we did not expect differences in laterality, all subjects were investigated at their right dorsal hand. 13 QST parameters were determined: cold and warm detection thresholds, thermal sensory limen (perception of changing temperatures from warm to cold and vice versa), paradoxical heat sensations (participant experiences cold as heat), cold and heat pain thresholds, mechanical detection and pain thresholds, mechanical pain sensitivity (sensitivity to pinprick stimuli), pressure pain threshold, vibration detection thresholds, mechanical allodynia (experience of pain during nonpainful stimulation) and the wind-up ratio (temporal pain summation $=$ ratio of pain ratings of a series of painful stimuli / pain ratings of a single painful stimulus). The room temperature was kept between 20 and $25^{\circ} \mathrm{C}$.

\section{Statistical analyses}

Statistical analyses were performed using the Equista software provided by the DFNS (http://www.neuro.med.tu-muenchen.de/dfns/arzt/qstform.html) and using IBM SPSS software version 20.0 (http://www-01.ibm.com/software/analytics/spss/). Initially, using Equista, raw values of both groups were log-transformed to establish normal distribution and mapped onto the distribution of the DFNS reference group $[8,10]$ consisting of 180 healthy subjects using z-transformation (z-score Participant $=((Q S T$ Participant - QST Reference) / standard deviation Reference))). This method assured comparability of QST results as z-scores were adjusted to sex, age and tested body site of the published reference group $[8,10]$ and has been used in a recent study [11]. 
A $z$-score $>0$ indicated high and a $z$-score $<0$ low sensitivity to the external stimulus applied. Z-scores exceeding $95 \%$ of the confidence interval of the reference group (+/1.96 standard deviation (SD)) are considered as pathologic. As there were no paradoxical heat sensations or allodynia in both groups, analyses proceeded with only 11 out of 13 QST parameters.

For between group analyses, mean z-scores were compared between GTS and control group using parametric student's t-test. Furthermore, group mean z-scores and individual z-scores were compared with the published reference data $[8,10]$ and analyzed with a view to neurobiological (topodiagnostic) mechanisms assessing, for example, the functioning of different types of nerve fibres (e.g. A delta, A beta or C fibers) or more central mechanisms such as sensitization [12]. Finally, z-scores were correlated with PUTS scores using Pearson's correlation coefficients.

\section{RESULTS}

14 GTS patients without relevant comorbidities (mean age 31.7+/-SD 7.8 years) and 14 healthy control subjects (32.7+/-7.8 years) matched by age $(t(26)=-0.339, p=0.737)$ and gender (each group: 2 female) were included in the study. Patient characteristics are given in Table 1 (see also supplementary table 1). All patients reported tic-related premonitory sensations as given in Table 1 .

\section{[please insert Table 1 here]}

Initial group comparisons of single QST parameters' mean z-scores revealed a significant difference for the cold detection threshold (CDT, $t(26)=4.175 ; p<0.001)$ even after Bonferroni correction with a gain of function for cold detection in GTS patients (mean z-score $0.61+/-$ SD 0.63 ) and a loss of function in healthy subjects (mean z- 
score $-0.68+/-$ SD 0.97$)$. However, CDT mean z-scores of both groups ranged within +/-1.96 SD of the DFNS reference group. All other QST parameters did not differ significantly between both groups (all $p>0.15$, see Figure 1 , part A and supplementary table 1). None of the patients or controls experienced allodynia or paradoxical heat sensation.

When analyzing individual CDT z-scores (see Figure 1, part B and C), apart from one marginally increased $z$-score in the GTS group $(z=2.05)$ and one marginally decreased $z$-score in the control group $(z=-1.99)$, all the other CDT $z$-scores ranged within $+/-$ 1.96 SD of the DFNS reference group (C). Thus CDT-scores in GTS can be classified as normal. Regarding individual analysis of the other QST parameters in comparison with the DFNS reference data, there were a few more "outliers" in the GTS group as compared to controls, but no consistent, pathologic QST pattern pointing towards a certain neurobiological mechanism such as peripheral nerve fiber dysfunction or peripheral / central sensitization.

\section{[please insert Figure 1 here]}

In the GTS group, PUTS scores did not correlate with any of the QST parameters (all $p>0.1)$.

\section{DISCUSSION}

Increased somatic sensitivity has been proposed as an explanation for the presence of abnormal sensory phenomena, such as tic-related premonitory sensations in GTS [3]. Focusing on the exteroceptive system we investigated this hypothesis by using 
QST as a comprehensive, established test battery assessing sensory thresholds in a sample of uncomplicated GTS adults and healthy controls matched for age and gender. Although we found group differences for cold detection (CDT) in direct group comparison, mean z-scores of both groups were within the range of the DFNS reference data. Therefore, the isolated CDT difference in conjunction with normal zscores for other thermal QST parameters and normal A-delta-fiber function (represented by e.g. pain to pinprick stimuli) cannot be regarded as pathological. There was no specific, pathologic QST pattern suggesting a defined neurobiological mechanism (e.g. peripheral nerve fiber dysfunction or peripheral / central sensitization) was identified. Furthermore, there was no relation between sensory thresholds within the GTS group and the trait intensity of premonitory sensations as determined with the PUTS.

Two previous studies examined sensory thresholds in GTS and reported conflicting findings [4, 7]. Importantly, the two studies employed different methods and tested no more than two sensory modalities. Using a well-established and standardized QST method, we here provide a systematic assessment for different exteroceptive somatosensory channels and show normal thresholds in GTS. The sensory profiling using QST further involves the testing of suprathreshold pain stimuli, which to our knowledge have also not been studied systematically in QTS. Our study does not reveal any differences in nociception in GTS patients. Also, different from both previous studies [4, 7], we restricted our study to uncomplicated GTS adults. Hence, the influence of the potential confounding effects of common GTS-related comorbidities on our findings is unlikely.

To our knowledge, the relation between mechanical and thermal sensory thresholds and premonitory sensations has not been studied before. Although all of our patients 
reported the presence of sensory phenomena (type and location were similar to Kwak et al. [2]), there was no correlation between QST parameters and premonitory sensations. Importantly, the sensory phenomena characteristic of GTS are always described as internal to the body itself, while the measures of QST are always based on activating somatosensory afferents by delivering an overt external stimulation. The attribution of sensory events to an internal or to an external source plays an important role in how those events are perceived [13]. We speculate that the distinction between internal and external sensory attribution may underlie the simultaneous presence of normal QST findings and abnormal sensory phenomena in the patient group. In particular, perceptual detection of external mechanical and thermo-mechanical stimuli does not seem to play an important role for the perception of premonitory sensations. Other perceptual sources, as for example interoception, might be more important in the awareness of sensory phenomena in GTS [5]. Also, other mechanisms such as altered central somatosensory processing including sensory gating dysfunction [7] possibly mediated by alterations in cortico-striato-thalamo-cortical and insular circuits [3] might further contribute to hypersensitivity and premonitory sensations in GTS.

One limitation of our study is the relatively small sample size resulting from the use of a well-selected adult GTS patient group without relevant neuropsychiatric comorbidities that is particularly rare in the clinical setting, but may represent only one end of the GTS spectrum. However, this approach allowed us to perform a detailed clinical assessment and comprehensive sensory threshold assessment free from confounders such as ADHD or OCD. Also, sensory profiles were obtained at a standardized location (dorsum of hand) which assured comparability with the DFNS reference group, but results could differ in body parts most affected by premonitory sensations or tics e.g. the face. It should be noted, however, that one third of our patients had reported premonitory sensations in their hands. Of note, in the previous 
study by Belluscio et al. all regions of the body were similarly sensitive to external stimuli and thresholds at the most affected body part did not differ from those in less affected body parts [4].

In summary, contrary to our hypothesis, no disease-specific, pathologic QST pattern was found in GTS patients. This indicates that other mechanisms e.g. interoceptive awareness or altered central processing might underlie clinically significant sensory abnormalities in GTS. 


\section{ACKNOWLEDGEMENTS}

We would like to thank all the patients investigated in this study for their support and

commitment. Thanks to Jan Vollert (University Medical Center Bochum) and Tina Mainka (University Medical Center Hamburg-Eppendorf) for their advice.

\section{CONFLICTS OF INTERESTS}

None.

\section{FUNDING}

This study was funded by the German Research Foundation (DFG MU1692/2-1).

\section{REFERENCE LIST}

[1] American Psychiatric Association (APA). Diagnostic and statistical manual of mental disorders DSM-IV-TR. 4 ed. Washington, DC: American Psychiatric Association; 2000.

[2] Kwak C, Dat Vuong K, Jankovic J. Premonitory sensory phenomenon in Tourette's syndrome. Mov Disord. 2003;18:1530-3.

[3] Jackson SR, Parkinson A, Kim SY, Schuermann M, Eickhoff SB. On the functional anatomy of the urge-for-action. Cognitive neuroscience. 2011;2:227-43.

[4] Belluscio BA, Jin L, Watters V, Lee TH, Hallett M. Sensory sensitivity to external stimuli in Tourette syndrome patients. Mov Disord. 2011;26:2538-43.

[5] Ganos C, Garrido A, Navalpotro-Gomez I, Ricciardi L, Martino D, Edwards MJ, et al. Premonitory urge to tic in tourette's is associated with interoceptive awareness. Movement disorders: official journal of the Movement Disorder Society. 2015. [Epub ahead of print] 
[6] Thomalla G, Siebner HR, Jonas M, Baumer T, Biermann-Ruben K, Hummel F, et al. Structural changes in the somatosensory system correlate with tic severity in Gilles de la Tourette syndrome. Brain : a journal of neurology. 2009;132:765-77.

[7] Puts NA, Harris AD, Crocetti D, Nettles C, Singer HS, Tommerdahl M, et al. Reduced GABAergic inhibition and abnormal sensory symptoms in children with Tourette syndrome. Journal of neurophysiology. 2015;114:808-17.

[8] Rolke R, Baron R, Maier C, Tolle TR, Treede RD, Beyer A, et al. Quantitative sensory testing in the German Research Network on Neuropathic Pain (DFNS): standardized protocol and reference values. Pain. 2006;123:231-43.

[9] Rössner V, Müller-Vahl K, Neuner I. PUTS - premonitory urge tics scale: Fragebogen für Kinder. In: Müller-Vahl K., editor. (Ed.), Tourette-Syndrom und andere Tic-Erkrankungen im Kindes- und Erwachsenenalter. Berlin, Germany: MWV Medizinische Wissenschaftliche Verlagsgesellschaft; 2010.

[10] Magerl W, Krumova EK, Baron R, Tolle T, Treede RD, Maier C. Reference data for quantitative sensory testing (QST): refined stratification for age and a novel method for statistical comparison of group data. Pain. 2010;151:598-605.

[11] Lotsch J, Dimova V, Hermens H, Zimmermann M, Geisslinger G, Oertel BG, et al. Pattern of neuropathic pain induced by topical capsaicin application in healthy subjects. Pain. 2015;156:405-14.

[12] Mucke M, Cuhls H, Radbruch L, Baron R, Maier C, Tolle T, et al. [Quantitative sensory testing]. Schmerz. 2014;28:635-46; quiz 47-8.

[13] Green BG. Temperature perception on the hand during static versus dynamic contact with a surface. Attention, perception \& psychophysics. 2009;71:1185-96. 